\title{
Knockdown of the prion gene expression by RNA interference in bovine fibroblast cells
}

\author{
Shaohua Wang $\cdot$ Xiaoqing Lv $\cdot$ Kun Zhang $\cdot$ \\ Tonghui Lin $\cdot$ Xiaofang Liu $\cdot$ Jing Yuan • \\ Yunping Dai $\cdot$ Ning $\mathbf{L i}$
}

Received: 20 September 2009/ Accepted: 2 October 2009

(C) Springer Science+Business Media B.V. 2009

\begin{abstract}
P R N P$ is the gene encoding prion protein whose misfolded and $\beta$-sheet-rich isoform is the infectious agent of transmissible spongiform encephalopathy (TSE). TSE, also called prion diseases, cause fatal neurodegenerative and transmissible disorders in human and animals. Among these diseases, bovine spongiform encephalopathy (BSE) has tremendous impact on economy and human health in the world. In the present study, we hypothesize suppression of the PRNP gene expression could raise resistance to BSE in cattle by using vectorbased small interfering RNA (siRNA) expression systems. Therefore, the objective was to screen effective DNAencoding short hairpin RNAs (shRNAs) which could knockdown the PRNP gene expression in bovine fibroblast cells. Human U6 promoter was employed to drive
\end{abstract}

S. Wang $\cdot$ X. Lv $\cdot$ K. Zhang $\cdot$ T. Lin $\cdot$ X. Liu $\cdot$ J. Yuan

Y. Dai $(\bowtie) \cdot$ N. Li $(\bowtie)$

State Key Laboratory for Agrobiotechnology, College

of Biological Science, China Agricultural University,

Yuanmingyuan West Road No. 2, Haidian District, Beijing

100193, People's Republic of China

e-mail: daiyunping@sina.com

N. $\mathrm{Li}$

e-mail: ninglcau@cau.edu.cn

S. Wang

e-mail: shwang510@gmail.com

Y. Dai · N. Li

Beijing Gene \& Protein Biotechnology Co., Ltd., Room 306,

Zhongding Building, Malianwa North Road No.158, Haidian

District, Beijing 100193, People's Republic of China

Present Address:

K. Zhang

Animal Molecular and Cellular Biology Program, Institute

of Food and Agricultural Sciences, University of Florida,

Gainesville, FL 32611, USA
shRNA transcription from the DNA vector, and seven shRNAs, that designed to target coding region and $3^{\prime}$ untranslated region of the PRNP gene, were selected. Four out of seven shRNAs tested were found to be effective in inhibiting the PRNP gene expression, and the most significant suppression level was as much as $62.9 \%$ evidenced by real-time RT-PCR. Furthermore, the protein abundance was obviously reduced compared to the control. Overall, the present study demonstrated that vectorbased siRNA expression systems is an efficient approach to knockdown the PRNP gene expression in bovine fibroblast cells and thereby provide donor cells for somatic cell nuclear cloning to produce cattle that is resistant to prion related diseases.

Keywords Prion - RNA interference - Bovine · Fibroblast cells $\cdot$ shRNA

\section{Introduction}

Misfolded isoform of the prion protein, named pathogenic prion protein $\left(\mathrm{PrP}^{\mathrm{Sc}}\right)$, is the infectious agent that cause transmissible spongiform encephalopathy (TSE) in human and animals [1] [In cattle, the TSE was usually called BSE (bovine spongiform encephalopathy) or mad cow disease.]. The cellular prion protein $\left(\operatorname{PrP}^{\mathrm{C}}\right)$, a cell membrane glycoprotein, is primarily detected in neuronal tissue [2] and may be involved in signal transduction [3, 4]. The conversion from normal $\operatorname{PrP}^{\mathrm{C}}$ to $\operatorname{PrP}^{\mathrm{Sc}}$ is recognized as the key event to develop prion disease based on the "protein-only" hypothesis [5]. It has been justified that $\operatorname{PrP}^{\mathrm{C}}$-deficient $\left(P R N P^{-1-}\right)$ mice are resistant to TSE and do not propagate infectious $\mathrm{PrP}^{\mathrm{Sc}}$ without apparent developmental or behavioral defects [6-9]. Therefore, suppression of 
endogenous $\mathrm{PrP}^{\mathrm{C}}$ expression could inhibit endogenous or exogenous $\operatorname{PrP}^{\mathrm{Sc}}$ formation and accumulation and thereby protect individuals from prion diseases.

Currently, cattle are recognized as potential bioreactor to produce human-use products besides milk and meat, and BSE in cattle may be a concern in the application of animal products for human use. Accordingly, it is prudent to produce human-use proteins in BSE-free cattle. During the past few years, gene targeting technology has been successfully applied to produce prion-resistant cattle [10, 11]. Within this strategy, bovine fibroblast cells were transfected with the PRNP gene targeting vector. After antibiotic selection, cells which undergo homologous recombination were used as donors to produce BSE-resistant cattle via nuclear transfer. However, it is technically complicating, time-consuming and costly since the inherent efficiency of the homologous recombination in somatic cells is extremely low.

RNA interference (RNAi), a conserved post-transcriptional gene silencing technology, provides a wide and powerful tool to specifically silence both endogenous protein-coding and exogenous pathogenic gene expression [12]. With RNAi, small interfering RNAs (siRNAs), which is processed from long double-stranded RNAs (dsRNAs) by the dsRNA endonuclease Dicer, can be bound to complementary mRNA and trigger specific mRNA degradation. In mammalian cells, synthetic siRNAs can be transferred into cells and induce transient gene silencing. However, the suppression effect lasts only several days because of the lack of siRNA amplification machinery in mammalian cells. Consequently, stable gene transfer and vector-based siRNA expression systems have been developed in order to resolve this problem. It has been found RNA polymerase III promoters, such as U6 or H1, could drive short hairpin RNAs (shRNAs) transcription from DNA templates, and these shRNAs subsequently are cleaved by the Dicer to produce siRNAs to introduce mRNA degradation in mammalian cells [13-16].

In the previous studies [17-20], knockdown of the prion gene by RNAi was usually carried out in transgenic mice or cell lines which transiently or stably expressed the prion protein. However, differences may exist between the transgenic PRNP mRNA and the wild type mRNA in terms of the length and the spatial structure so as to affect screening of the effective shRNAs. Therefore, it is necessary to perform shRNAs screen in cells which naturally express the prion gene.

Here in the present study, we detected the prion protein gene expression in bovine fibroblast cells and established a robust method to screen effective shRNAs to knockdown the prion gene expression in bovine fibroblast cells.

\section{Materials and methods}

Construction of the shRNA expression vectors

Invitrogen's RNAi Designer (https://rnaidesigner.invitrogen. com/rnaiexpress/design.do) was utilized to design shRNA sequences against bovine PRNP mRNA sequence (GenBank accession No.NM_181015) and coding domain sequence (GeneBank accession No.AY247262). One scrambled shRNA control was specifically designed. Two complementary single-stranded DNA oligonucleotides of each shRNA were chemically synthesized by Shanghai Sangon Biotechnology Co. Ltd (China) and then annealed to generate double-stranded oligonucleotides (ds oligo). Subsequently annealed ds oligo was cloned into BamHI/HindIII linearized pGenesil-1 vector (Wuhan Genesil Biotechnology Co. Ltd., China) which contains U6 promoter to transcribe shRNA. DNA sequencing was performed to verify the sequence of the ds oligo insert.

RNA isolation and reverse transcription

Total RNA were isolated from bovine fibroblast cells with Qiagen RNeasy ${ }^{\circledR}$ Mini kit (Qiagen, Germany) according to the manufacturer's instructions. The integrity of isolated total RNA was analyzed by $0.7 \%$ agarose gel electrophoresis. $2-5 \mu \mathrm{g}$ total RNA were reverse transcribed by SuperScript ${ }^{\mathrm{TM}}$ III Reverse Transcriptase (Invitrogen, USA) with oligo $(\mathrm{dT})_{18}$ primers.

Construction of the psiCHECK ${ }^{\mathrm{TM}}-2-P R N P$ vectors

The bovine PRNP mRNA was amplified by RT-PCR with primers listed in Table 1. One $965 \mathrm{bp}$ fragment spans exon 2 and exon3, and the other $767 \mathrm{bp}$ fragment spans the $3^{\prime}$ untranslated region. One microliter of undiluted cDNA from the RT reaction was employed as template for each $25 \mu \mathrm{l}$ PCR reaction. The PCR condition was: $94^{\circ} \mathrm{C}$ for $5 \mathrm{~min} ; 35$ cycles of $94^{\circ} \mathrm{C}$ for $30 \mathrm{~s}, 58^{\circ} \mathrm{C}$ for $30 \mathrm{~s}$ and $72^{\circ} \mathrm{C}$ for $50 \mathrm{~s} ; 72^{\circ} \mathrm{C}$ for $10 \mathrm{~min}$. The PCR products were purified and digested by XhoI (TaKaRa, Japan) and NotI (TaKaRa, Japan), and finally cloned into XhoI/NotI linearized psiCHECK $^{\mathrm{TM}}$-2 vector (Promega, USA). DNA sequencing was conducted to verify the sequence.

Cell culture and transfection

Bovine fibroblast cells and human embryonic kidney 293 cells (HEK-293) were cultured in Dulbecco's Modified Eagle Medium (DMEM; Gibco BRL, USA) supplemented with $10 \%$ fetal bovine serum (FBS; HyClone, USA) at $37.5^{\circ} \mathrm{C}$ under $5 \% \mathrm{CO}_{2}$., Cells were seeded in 24-well plates or 6-well plates, and cultured for one day to obtain 70-90\% 
Table 1 PCR primers used in the present study

\begin{tabular}{|c|c|c|c|c|c|}
\hline Primer name & Gene & NCBI accession No. & Location & Primer sequence $\left(5^{\prime} \rightarrow 3^{\prime}\right)$ & $\begin{array}{l}\text { Length } \\
\text { of products }\end{array}$ \\
\hline psiCHECK-F1 ${ }^{\text {a }}$ & $P R N P$ & NM_181015 & Exon 2 & TGACTCGAGTCAACCAAGCCGAAGCATCTG & 965 bp \\
\hline psiCHECK-R $1^{\mathrm{a}}$ & $P R N P$ & NM_181015 & Exon 3 & TAT $\boldsymbol{G} \boldsymbol{C} \boldsymbol{G} \boldsymbol{G} \boldsymbol{C} \boldsymbol{C} \boldsymbol{C G A A A T G A G A C A C C A C C A C T A C ~}$ & \\
\hline psiCHECK-F2 ${ }^{\mathrm{a}}$ & $P R N P$ & NM_181015 & $3^{\prime} \mathrm{UTR}$ & TCACTCGAGCGACTGAGTGACTGAACTGAG & $767 \mathrm{bp}$ \\
\hline psiCHECK-R2 ${ }^{\mathrm{a}}$ & $P R N P$ & NM_181015 & $3^{\prime}$ UTR & ATA $\boldsymbol{G} \boldsymbol{C} \boldsymbol{G} \boldsymbol{G} \boldsymbol{C} \boldsymbol{G} \boldsymbol{C}$ TGAACCTTTTAGCCAACCCAG & \\
\hline RT-F ${ }^{b}$ & $P R N P$ & NM_181015 & Exon 2 & AAATCCAACTTGAGCTGAATC & $843 \mathrm{bp}$ \\
\hline RT-R ${ }^{b}$ & $P R N P$ & NM_181015 & Exon 3 & GGTTGCCCCTATCCTACTATG & \\
\hline $\mathrm{Q}-83 \mathrm{~F}^{\mathrm{C}} *$ & $P R N P$ & NM_181015 & Exon 2 & GTTTCAACCAAGCCGAAGCA & $134 \mathrm{bp}$ \\
\hline Q-195R $\mathrm{R}^{\mathrm{c}}$ & $P R N P$ & NM_181015 & Exon 3 & CCAGGATCCAACTGCCTATGTG & \\
\hline GAPDH-F ${ }^{\mathrm{d} *}$ & $G A P D H$ & NM_001034034 & Exon 11 & CGACTTCAACAGCGACACTCAC & $120 \mathrm{bp}$ \\
\hline GAPDH-R ${ }^{\mathrm{d} *}$ & $G A P D H$ & NM_001034034 & Exon 12 & CCCTGTTGCTGTAGCCAAATTC & \\
\hline
\end{tabular}

${ }^{a}$ Primers used for cloning fragment of the PRNP cDNA to construct the psiCHECK ${ }^{\mathrm{TM}}-2-P R N P$ vectors

${ }^{\mathrm{b}}$ Primers used for RT-PCR and semi-quantitative RT-PCR analysis of the PRNP mRNA expression in bovine fibroblast cells

c Primers used for real-time RT-PCR analysis of the PRNP mRNA expression in bovine fibroblast cells

${ }^{\mathrm{d}}$ Primers for housekeeping genes used for standardization of real-time and semi-quantitative RT-PCR

* Primers designed by Primer Express Software (Applied Biosystems)

CTCGAG, XhoI restriction site; $\boldsymbol{G C G G C C G C}$, NotI restriction site

confluence. Then, transfection was performed using Lipofectamine $^{\mathrm{TM}} 2000$ reagent (Invitrogen, USA) according to the manufacturer's instructions.

Screening of the effective shRNAs using

double-luciferase detection system

HEK-293 cells plated in 24-well plates were grown to $70-90 \%$ confluence. Individual wells were transfected with $500 \mathrm{ng}$ of plasmid DNA (210 ng of the psiCHECK- $2^{\mathrm{TM}}$ PRNP plasmid and $290 \mathrm{ng}$ of the pGenesil-1 shRNA expression vector). Sixty hours post-transfection, Renilla and firefly luciferase activities were measured with the Dual-Glo ${ }^{\mathrm{TM}}$ Luciferase Assay System (Promega, USA). To evaluate the suppression levels, the ratio of Renilla/Firefly in cells transfected with each test shRNA were compared to that in cells transfected with scrambled shRNA.

Semi-quantitative and real-time RT-PCR

Sixty hours after bovine fibroblast cells transfected with the pGenesil-1 shRNA expression vectors, cells were sorted by fluorescence-activated cell sorting (FACS; DakoCytomation, Inc.,Ft. Collins, USA), and GFP positive $\left(\mathrm{GFP}^{+}\right)$ cells were collected for total RNA isolation. Semi-quantitative and real-time RT-PCR were used to detect the PRNP mRNA expression level. The semi-quantitative RT-PCR products covered full length of the PRNP coding region, and the PCR condition was: $94^{\circ} \mathrm{C}$ for $5 \mathrm{~min}$; 35 cycles of $94^{\circ} \mathrm{C}$ for $30 \mathrm{~s}, 56^{\circ} \mathrm{C}$ for $30 \mathrm{~s}$ and $72^{\circ} \mathrm{C}$ for $50 \mathrm{~s} ; 72^{\circ} \mathrm{C}$ for 10 min. Real-time RT-PCR was performed using an ABI
Prism $^{\circledR}$ 7900HT Sequence Detection System (Applied Biosystems, USA). A standard PCR protocol was used for all primers: $50^{\circ} \mathrm{C}$ for $2 \mathrm{~min} ; 95^{\circ} \mathrm{C}$ for $10 \mathrm{~min} ; 40$ cycles of $95^{\circ} \mathrm{C}$ for $20 \mathrm{~s}, 60^{\circ} \mathrm{C}$ for $1 \mathrm{~min}$; followed by $95^{\circ} \mathrm{C}$ for $15 \mathrm{~s}$, $60^{\circ} \mathrm{C}$ for $15 \mathrm{~s}, 95^{\circ} \mathrm{C}$ for $15 \mathrm{~s}$ to draw dissociation curve. Relative expression levels of the PRNP gene were normalized to GAPDH expression levels. All primers were shown in Table 1.

\section{Western blot}

Sixty hours after bovine fibroblast cells transfected with the pGenesil-1 shRNA expression vectors, cells were sorted by FACS, and $\mathrm{GFP}^{+}$cells were collected. These $\mathrm{GFP}^{+}$cells and untransfected bovine fibroblast cells were washed twice with Dulbecco's Phosphate-Buffered Saline (DPBS; Gibco BRL, USA), and then lysed with immunoprecipitation(IP) cell lysis buffer $\left(100 \mu 1 / 10^{6}\right.$ cells; Beyotime Institute of Biotechnology, China). After $30 \mathrm{~min}$ incubation on ice, the cell lysates were centrifuged at $4^{\circ} \mathrm{C}$, $13,000 \times g$ for $5 \mathrm{~min}$. The supernatants were collected for western analysis. Protein concentration of each sample was measured by BCA Protein Assay Kit (Beyotime Institute of Biotechnology, China) following the manufacturer's instructions. Protein samples were separated by $12 \%$ SDSPAGE gel and transferred to PVDF (Polyvinylidene Fluioride) membranes (GE Healthcare, UK). For the PRNP gene expression detection, the membranes were incubated with rabbit anti-prion protein polyclonal antibody (Abcam Inc., USA) at room temperature for $2 \mathrm{~h}$ and stained by the secondary HRP-conjugated goat anti-rabbit IgG antibody 
(Santa Cruz Biotechnology, Inc., USA) for $1 \mathrm{~h}$. Then, the membranes were visualized using SuperSignal West Dura Extended Duration Substrate (Pierce Manufacturing, Inc., USA). $\beta$-actin was used as internal positive control in this experiment.

\section{Statistical analysis}

The real-time RT-PCR data were analyzed by the comparative $C_{T}$ method. Values were shown as means \pm SEM.

\section{Results}

Detection of the prion protein gene expression in bovine fibroblast cells

RT-PCR analysis indicated the PRNP gene was transcriptional active in bovine fibroblast cells (Fig. 1a, b), and the $26 \mathrm{kDa}$, non-glycosylated $\mathrm{PrP}^{\mathrm{C}}$, could be detected in the protein lysate from bovine fibroblast cells (Fig. 1c). As shown in Fig. 1d, different amount of the protein lysate from bovine fibroblast cells was analyzed, and the prion protein could be clearly seen in $5 \mu \mathrm{g}$ total protein lysate.

Screening of the effective shRNAs using double-luciferase detection system

Seven shRNAs, five from the PRNP coding domain sequence and two from the PRNP mRNA $3^{\prime}$ untranslated ( $3^{\prime}$ UTR) region, were designed with Invitrogen's RNAi Designer. To facilitate screening of the effective shRNAs against the prion protein, a PRNP mRNA fragment was subcloned into downstream of the Renilla luciferase coding region within the psiCHECK- $2^{\mathrm{TM}}$ vector. The psiCHECK2-PRNP expression vector would produce a fusion mRNA containing the coding sequence of the Renilla luciferase

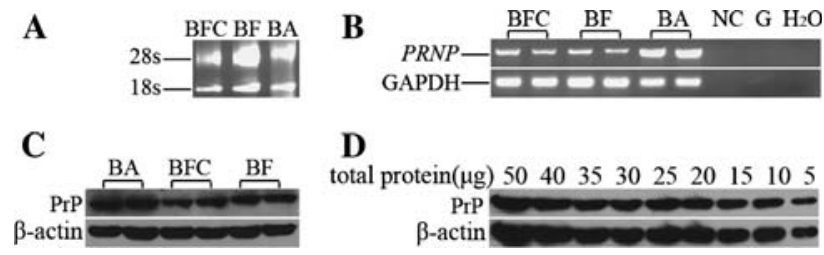

Fig. 1 Detection of the prion gene expression in bovine fibroblast cells. $B F C$ bovine fibroblast cells, $B F$ brain of bovine fetus, $B A$ brain of adult cattle. a Analysis of the total RNA by agarose gel electrophoresis. b RT-PCR analysis of the PRNP mRNA expression in bovine fibroblast cells. $N C$ negative control of reverse transcript, $G$ bovine genomic DNA. The prion gene is expressed in bovine fibroblast cells. $\mathbf{c}$ Western blot analysis of the prion protein expression in bovine fibroblast cells. $\mathbf{d}$ Western blot analysis of different amount of total protein from bovine fibroblast cells. Prion protein could be detected in $5 \mu \mathrm{g}$ total protein lysate by western blot

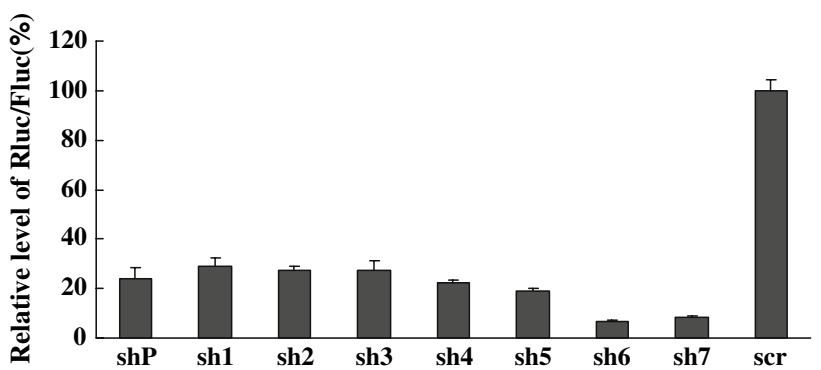

Fig. 2 Screening of the effective shRNAs using double-luciferase detection system in HEK-293 cells. The psiCHECK-2-PRNP and shRNA expression vectors were cotransfected with HEK-293 cells. The suppression level of different shRNA targeting to PRNP was indicated by the Renilla activity normalized to firefly luciferase activity. All data are presented as the percentage of Rluc/Fluc compared with the control unrelated shRNA, and the value are an average of four independent experiments, and actual percentages and standard deviations for the shRNAs are as follows: shP $24.06 \pm 4.46 \%$, sh1 $29.05 \pm 3.55 \%, \quad$ sh2 $27.16 \pm 1.74 \%$, sh3 $27.40 \pm 3.65 \%, \quad \operatorname{sh} 422.16 \pm 1.02 \%, \quad$ sh5 $19.10 \pm 1.11 \%, \quad$ sh6 $6.94 \pm 0.23 \%$, sh7 $8.14 \pm 0.58 \%$, scr $100 \pm 4.53 \%$. shP shRNA against Renilla luciferase, shl-7 seven shRNAs at different target sites to PRNP mRNA, scr scrambled shRNA control

followed by a non-translated PRNP mRNA as well as the Firefly luciferase which acts as a means of normalization. Following HEK-293 cells co-transfected with the psiCHECK-2-PRNP and the shRNA expression vectors, the Renilla/Firefly luciferase activity ratio could indicate the suppression degree of the PRNP mRNA expression. As shown in Fig. 2, all of the seven shRNAs could efficiently reduce Renilla luciferase expression. This result demonstrated that human U6 promoter could effectively drive shRNA transcription in HEK-293 cells and all of the shRNAs were effective to inhibit the PRNP mRNA expression.

Suppression of the prion protein gene expression in bovine fibroblast cells

Transient transfection of the bovine fibroblast cells was conducted to detect the suppression of the prion gene expression by each shRNA expression vector. As the transfection efficiency of the bovine fibroblast cells was low, around $25 \%$, in this research, FACS was used to enrich cells which were transfected with the shRNA expression vectors, and the following detection was carried out in these cells. Out of the seven shRNA expression vectors, four resulted in obvious reductions in the PRNP mRNA expression level compared to the non-transfected cells (Fig. 3a). The PRNP mRNA expression level was reduced by $35.7,60.2,55.1$ and $62.9 \%$, respectively (Fig. 3b).

It was also shown that the prion protein level was reduced by the shRNA transfection when $5 \mu \mathrm{g}$ total protein lysate was analyzed (Fig. 3c). 

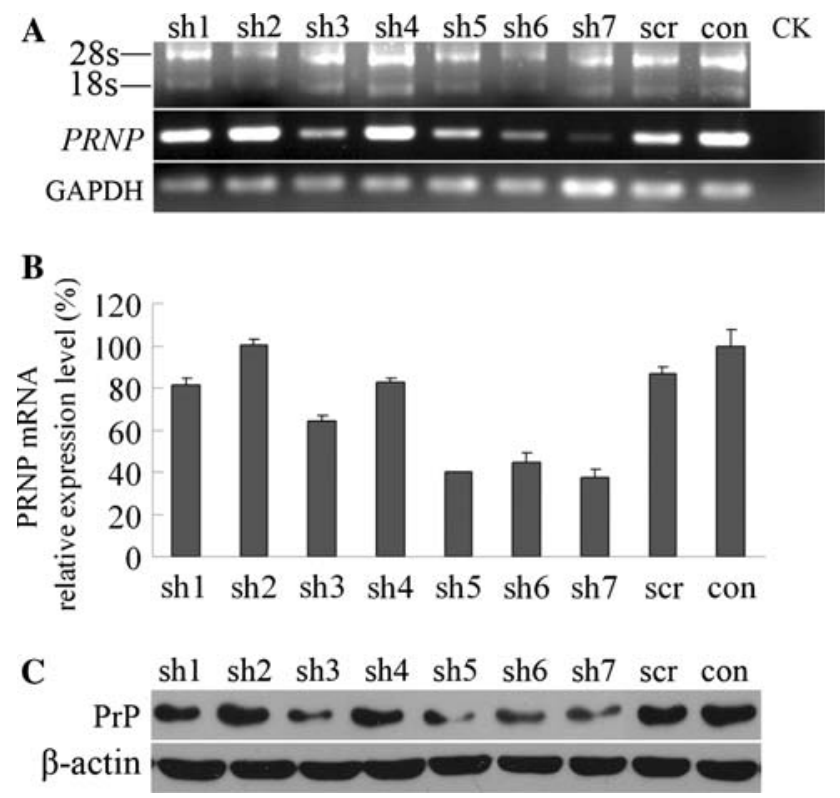

Fig. 3 Suppression of the prion protein gene expression by different shRNA expression vectors in bovine fibroblast cells. sh1-7 seven shRNAs at different target sites to the PRNP mRNA, scr scrambled shRNA control, con non-transfected bovine fibroblast cells, $C K$ bovine genome control. a Semi-quantitative RT-PCR analysis of the PRNP mRNA expression level. Total RNA isolated from bovine fibroblast cells transfected with different shRNAs was analyzed by agarose gel electrophoresis. After reverse transcription, GAPDH was amplified as internal control. b Real-time RT-PCR analysis of the $P R N P$ mRNA expression level in bovine fibroblast cells transfected with different shRNA expression vectors. The PRNP mRNA expression was normalized to GAPDH mRNA expression and denoted as a $P R N P / G A P D H$ ratio. Data from three independent experiments, and the actual percentages and standard deviations for the shRNAs are as follow: sh1 $81.57 \pm 2.88 \%$, sh2 $100.35 \pm 2.70 \%$, sh3 $64.28 \pm$ $2.86 \%$, sh4 $82.55 \pm 2.16 \%$, sh5 $39.76 \pm 0.46 \%$, sh6 $44.89 \pm$ $4.55 \%$, sh7 $37.14 \pm 4.23 \%$, scr $87.76 \pm 3.20 \%$, con $100 \pm 7.67 \%$. c Western blot analysis of the prion protein expression level in bovine fibroblast cells transfected with different shRNA expression vectors. Amount of total protein applied was $5 \mu \mathrm{g}$

\section{Discussion}

In the present study, we used DNA vector-based siRNA expression system to silence bovine PRNP gene expression not only in HEK-293 cells but also bovine fibroblast cells. Human U6 promoter was used to drive siRNA expression from the DNA templates and seven target sites were designed. Finally, we found four effective target sites that could down-regulate the prion gene expression in bovine fibroblast cells.

A fragment of the bovine PRNP mRNA sequence was inserted into the Renilla luciferase mRNA downstream region, thereby, a fusion mRNA containing the coding sequence of the Renilla luciferase followed by a nontranslated PRNP mRNA was transcribed after the transfection of the psiCHECK-2-PRNP expression vector. If the siRNA transcribed from the DNA vector could bind to the non-translated PRNP mRNA of the fusion mRNA and induce mRNA degradation, the coding sequence of the Renilla luciferase was degraded simultaneously. So the ratio of the expression level of Renilla luciferase, which is normalized by the firefly luciferase expression level, could indicate the silencing efficiency of individual target sites. As shown in Fig. 2, all of the seven siRNAs displayed almost the same inhibition degree compared with the siRNA against Renilla luciferase. This result suggested that the human U6 promoter could effectively drive siRNA transcribed from the DNA templates in HEK-293 cells.

In bovine fibroblast cells, the efficiency of cell transfection was around $25 \%$. Therefore, after the transfection of the shRNA expression vectors, FACS was used to collect positive cells which would express siRNAs. And then semi-quantitative and real-time RT-PCR indicated four out of seven target sites had reduced $35.7-62.9 \%$ of the PRNP mRNA expression. This was different from the double luciferase detection analysis. In the psiCHECK-2-PRNP expression vector, the length of PRNP mRNA was no more than $1 \mathrm{~kb}$, and the transcribed fusion mRNA may not form spatial structure. Transcribed siRNAs could easily bind to the complementary mRNA sequence and trigger RNAi process. In bovine fibroblast cells, the endogenous PRNP mRNA may have complicated spatial structure and this structure may block siRNA binding to the homology mRNA sequence which prevented RNAi process. Therefore, with the utilization of the double luciferase detection system, the effective target sites may not work well in vivo as in vitro, and further examination is needed.

In conclusion, four out of seven designed target sites had reduced the prion gene expression in bovine fibroblast cells, and two of them were at $3^{\prime}$ UTR region of the PRNP mRNA. Due to lower cost, vector-based shRNA expression approaches can be broadly applied in creating donor cells to produce prion diseases-resistant large animals in the future.

Acknowledgments We thank Mr. Fangrong Ding and Song Li for help in cell culture. This research was supported by the National High-tech Research and Development Program of China (Grant No. 2007AA02Z168).

\section{References}

1. Prusiner SB (1998) Prions. Proc Natl Acad Sci USA 95(23): 13363-13383

2. Tichopad A, Pfaffl MW, Didier A (2003) Tissue-specific expression pattern of bovine prion gene: quantification using realtime RT-PCR. Mol Cell Probes 17(1):5-10

3. Martins VR, Brentani RR (2002) The biology of the cellular prion protein. Neurochem Int 41(5):353-355

4. Ermonval M, Mouillet-Richard S, Codogno P, Kellermann O, Botti J (2003) Evolving views in prion glycosylation: functional and pathological implications. Biochimie 85(1-2):33-45 
5. Prusiner SB (1991) Molecular biology of prion diseases. Science 252(5012):1515-1522

6. Bueler H, Fischer M, Lang Y, Bluethmann H, Lipp HP, De Armond SJ, Prusiner SB, Aguet M, Weissmann C (1992) Normal development and behaviour of mice lacking the neuronal cellsurface PrP protein. Nature 356(6370):577-582

7. Prusiner SB, Groth D, Serban A, Koehler R, Foster D, Torchia M, Burton D, Yang SL, De Armond SJ (1993) Ablation of the prion protein $(\mathrm{PrP})$ gene in mice prevents scrapie and facilitates production of anti-PrP antibodies. Proc Natl Acad Sci USA 90(22): 10608-10612

8. Manson JC, Clarke AR, Hooper ML, Aitchison L, McConnell I, Hope J (1994) 129/Ola mice carrying a null mutation in PrP that abolishes mRNA production are developmentally normal. Mol Neurobiol 8(2-3):121-127

9. Bueler H, Aguzzi A, Sailer A, Greiner RA, Autenried P, Aguet M, Weissmann C (1993) Mice devoid of PrP are resistant to scrapie. Cell 73(7):1339-1347

10. Hirata RK, Xu C, Dong R, Miller DG, Ferguson S, Russell DW (2004) Efficient PRNP gene targeting in bovine fibroblasts by adeno-associated virus vectors. Cloning Stem Cells 6(1):31-36

11. Richt JA, Kasinathan P, Hamir AN, Castilla J, Sathiyaseelan T, Vargas F, Sathiyaseelan J, Wu H, Matsushita H, Koster J, Kato S, Ishida I, Soto C, Robl JM, Kuroiwa Y (2007) Production of cattle lacking prion protein. Nat Biotechnol 25(1):132-138

12. Elbashir SM, Harborth J, Lendeckel W, Yalcin A, Weber K, Tuschl T (2001) Duplexes of 21-nucleotide RNAs mediate RNA interference in cultured mammalian cells. Nature 411(6836): 494-498
13. Sui G, Soohoo C, Affar el B, Gay F, Shi Y, Forrester WC, Shi Y (2002) A DNA vector-based RNAi technology to suppress gene expression in mammalian cells. Proc Natl Acad Sci USA 99(8):5515-5520

14. Miyagishi M, Taira K (2002) U6 promoter-driven siRNAs with four uridine $3^{\prime}$ overhangs efficiently suppress targeted gene expression in mammalian cells. Nat Biotechnol 20(5):497-500

15. Lee NS, Dohjima T, Bauer G, Li H, Li MJ, Ehsani A, Salvaterra P, Rossi J (2002) Expression of small interfering RNAs targeted against HIV-1 rev transcripts in human cells. Nat Biotechnol 20(5):500-505

16. Paul CP, Good PD, Winer I, Engelke DR (2002) Effective expression of small interfering RNA in human cells. Nat Biotechnol 20(5):505-508

17. Tilly G, Chapuis J, Vilette D, Laude H, Vilotte JL (2003) Efficient and specific down-regulation of prion protein expression by RNAi. Biochem Biophys Res Commun 305(3):548-551

18. Pfeifer A, Eigenbrod S, Al-Khadra S, Hofmann A, Mitteregger G, Moser M, Bertsch U, Kretzschmar H (2006) Lentivector-mediated RNAi efficiently suppresses prion protein and prolongs survival of scrapie-infected mice. J Clin Invest 116(12):3204-3210

19. Sutou S, Kunishi M, Kudo T, Wongsrikeao P, Miyagishi M, Otoi T (2007) Knockdown of the bovine prion gene PRNP by RNA interference (RNAi) technology. BMC Biotechnol 7:44

20. Gallozzi M, Chapuis J, Le Provost F, Le Dur A, Morgenthaler C, Peyre C, Daniel-Carlier N, Pailhoux E, Vilotte M, Passet B, Herzog L, Beringue V, Costa J, Tixador P, Tilly G, Laude H, Vilotte JL (2008) Prnp knockdown in transgenic mice using RNA interference. Transgenic Res 17(5):783-791 